\title{
ARTICLE
}

Cite this: DOI: $10.1039 / \times 0 \times x 00000 x$

\section{A Label-Free Microfluidic Assay to quantitatively study antibiotic diffusion through lipid membranes}

\author{
J. Cama*a, C. Chimerel ${ }^{* a}$, S. Pagliara ${ }^{a}$, A. Javer ${ }^{a}$ and U. F. Keyser ${ }^{a}$
}

Received 00th January 2012, Accepted 00th January 2012

DOI: $10.1039 / \times 0 \times x 00000 x$

www.rsc.org/

\begin{abstract}
With the rise in antibiotic resistance amongst pathogenic bacteria, the study of antibiotic activity and transport across cell membranes is gaining widespread importance. We present a novel, label-free microfluidic assay that quantifies the permeability coefficient of a broad spectrum fluoroquinolone antibiotic, norfloxacin, across lipid membranes using the UV autofluorescence of the drug. We use giant lipid vesicles as highly controlled model systems to study the diffusion through lipid membranes. Our technique directly determines the permeability coefficient without requiring the measurement of the partition coefficient of the antibiotic.
\end{abstract}

\section{Introduction}

Antibiotic resistance in bacteria has developed into one of the world's foremost public health concerns ${ }^{1,2}$. The lack of new drugs combined with the rapid spread of resistant organisms poses a tremendous challenge for medical research and practice in the $21^{\text {st }}$ century. Antibiotic resistance develops in a number of ways: the lipid membrane can act as a barrier preventing the accumulation of the drug in the cell, the drugs might be enzymatically decomposed, the drug targets can be modified; in many cases multiple mechanisms are active simultaneously ${ }^{3,4}$. A better understanding of drug transport across lipid membranes is highly desirable and could facilitate the development of the next generation of antibiotics.

In our present drive towards miniaturisation and cost effectiveness in experimental science, microfluidics has emerged as a powerful tool. The small sample amounts required, high throughput capabilities and controlled microenvironments involved offer many advantages for the development of drug assays ${ }^{5,6,7,8,9,10}$. In this work, we develop a novel microfluidic technique to quantitatively examine the passive diffusion of antibiotics across lipid membranes. As a proof-of-principle, we study the passive diffusion of the broad spectrum fluoroquinolone antibiotic norfloxacin across vesicle lipid membranes using its autofluorescence ${ }^{11}$ in the UV. Norfloxacin targets the enzymes DNA gyrase and topoisomerase IV, disrupting DNA supercoiling and hence cell division ${ }^{12}$. Its accumulation within bacterial cells is therefore necessary for its antimicrobial activity. Previously, the accumulation of quinolones inside bacteria has been studied using radiolabelling ${ }^{13}$, bioassays ${ }^{14}$ and fluorimetry ${ }^{15}$, but it has been difficult to compare the published data due to differences in experimental conditions used ${ }^{16}$. These techniques do not separate the contributions to permeability from active and passive transport processes, and are further experimentally quite involved. 
a)

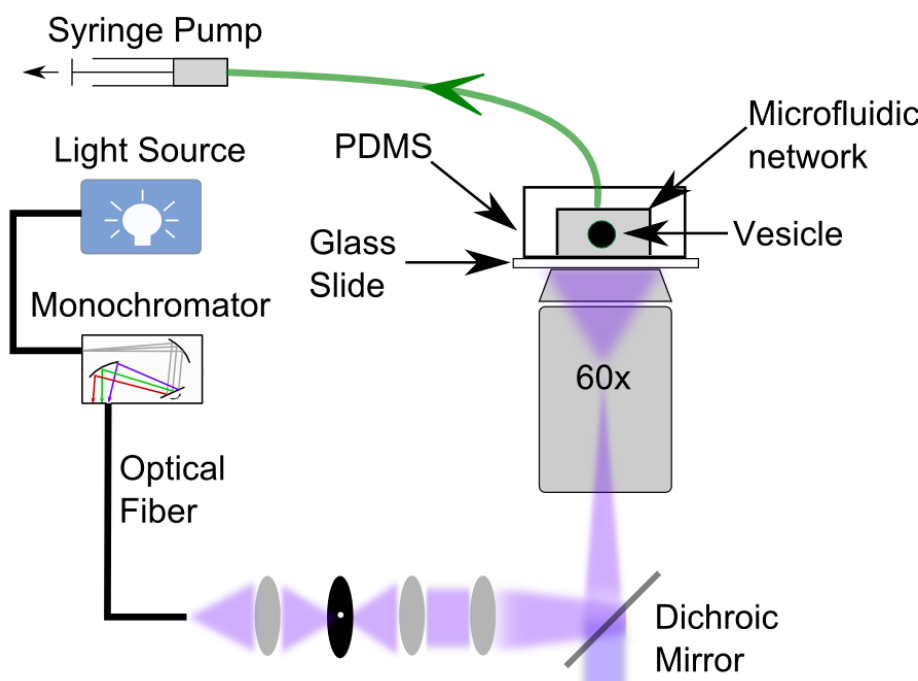

b)

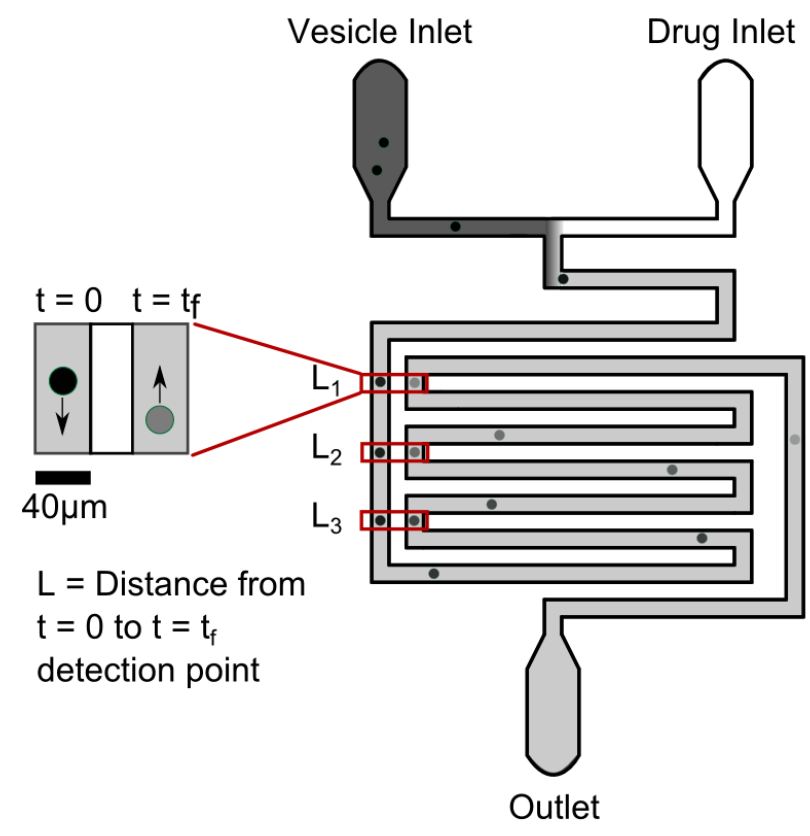

Figure 1a. Schematic of Optical Setup. Broadband white light is passed through a monochromator which selects the fluorescence excitation wavelength $(340 \mathrm{~nm})$. The excitation light is directed onto the microfluidic device and the emitted fluorescent radiation is focussed onto an EMCCD camera. Suction is applied at the outlet of the microfluidic network with a syringe pump. b. Schematic of microfluidic network. Lipid vesicles are exposed to a uniform concentration of the drug along the network. The $t=0$ point is chosen after the drug has equilibrated across the channel width. Vesicles at $t=0$ and later time points are observed in the same field of view. The two observation points are a length $L$ apart along the network. As vesicles progress along the network, if the drug is permeable, the fluorescence intensity inside the vesicles increases.

In our assay, we chose giant unilamellar vesicles as model systems, concentrating on passive transport in order to develop a better physical understanding of the interaction of the drug with a pure lipid membrane. It is estimated that between 8095\% of commercial drugs are absorbed primarily by passive diffusion ${ }^{17,18}$, and thus an assay to quantify permeability coefficients due to passive diffusion would be of great interest to the pharmaceutical industry. It has previously been demonstrated that giant vesicles are stable in microfluidic flows, and show potential for membrane transport studies 9 . Furthermore, small (diameter $\sim 180 \mathrm{~nm}$ ), fixed vesicles containing a reporter complex have been used to study drug permeation in a microfluidic environment ${ }^{10}$. Our technique, however, is label-free; vesicles flow through channels containing norfloxacin in a microfluidic network such that they can be observed at different locations and compared simultaneously with vesicles in their initial state. Determination of the norfloxacin fluorescence within the vesicles as they accumulate the drug over time allows us to calculate the permeability coefficient of the drug directly in an effective and simple manner.

The experiment is fast, uses small volumes $(\sim 10-100 \mu \mathrm{l})$ and can investigate individual vesicles in a label free manner. In contrast to traditional black lipid membrane techniques ${ }^{19}$, vesicles are more suitable cell mimics, and the advantage of using vesicles is that the same technique can then be adapted for cell use. This affords us the possibility of investigating active transport as well using suitable cell models. Furthermore, our assay directly evaluates the permeability coefficient without requiring information about the drug's partition coefficient; fluoroquinolone partition coefficients reported in the literature vary over a large range ${ }^{14,20,21}$ leading to a wide range of permeability values. A direct measurement of drug permeability coefficients is therefore highly desirable.

\section{Experimental details}

\section{Materials and methods}

Unless otherwise stated, all lenses and mirrors were obtained from Thorlabs UK and all chemicals from Sigma Aldrich UK.

\section{Optical Setup}

The optical setup is a custom built UV epifluorescence microscope described schematically in Figure 1a. The output from a broadband white light source (EQ99FC, Energetiq, USA) is passed to a monochromator (Monoscan 2000, OceanOptics) whereupon we select the desired excitation wavelength $(340 \mathrm{~nm})$ and direct it onto the microfluidic device via a Köhler illumination pathway and a suitable dichroic 
mirror (DLHS UV 351-355, Qi-Optiq, Germany). The objective used is a $60 \times$ water immersion UPLSAPO Olympus objective (NA 1.2). The emitted fluorescent light passes through the dichroic and is focussed onto the detector, an EMCCD camera (Evolve 512, Photometrics - exposure time $2 \mathrm{~ms}$, bin 2, EM gain 100, frame rate $65 \mathrm{fps}$ ) via a tube lens and a mirror. All lenses used (apart from the objective) are made from UV fused silica to optimise UV transmission and the optical fibers are UV grade (OceanOptics). The camera is controlled using the opensource software $\mu$ Manager $1.4^{22}$.

\section{Microfluidic Chip Design and Fabrication}

The microfluidic chip (schematic - Figure 1b) was constructed using standard photo- and soft lithography techniques ${ }^{23,24}$. The mask was designed in AutoCAD and printed on emulsion film (JD Photo-Tools UK). The design is attached in the electronic supplementary materials. SU8 3050 (Chestech UK) photoresist was spin coated (SCS Spincoat G3P-8, 7s at $500 \mathrm{rpm}$ followed by $30 \mathrm{~s}$ at $3,000 \mathrm{rpm}$ ) onto a $\mathrm{Si}$ wafer (Microchemicals, Germany) and then baked on a hot plate $\left(96{ }^{\circ} \mathrm{C}\right.$ for $\left.20 \mathrm{mins}\right)$. After aligning the film mask on the wafer, it was exposed to UV (the UV lamp was an OAI system with an output power of $200 \mathrm{~W}$ ) for 15 seconds, post-baked for 6-7 mins at $96{ }^{\circ} \mathrm{C}$ and then developed in Propylene glycol monomethyl ether acetate (PGMEA) for approximately $10 \mathrm{mins}$. This was then rinsed with isopropanol and blow dried with $\mathrm{N}_{2}$. A negative replica of the mold was obtained using Sylgard 184 Polydimethylsiloxane (PDMS- from Dow Corning); a 9:1 ratio of elastomer:curing agent PDMS mixture was poured onto the Si mold and cured for $55 \mathrm{mins}$ at $60{ }^{\circ} \mathrm{C}$ in an oven. The fabricated device (Figure 1b) has a network of microfluidic channels that are $40 \mu \mathrm{m}$ wide and $50 \mu \mathrm{m}$ high; the total length of the network from the $\mathrm{T}$ junction to the outlet reservoir is approximately $380 \mathrm{~mm}$. There is also a network of filter pillars in the inlet reservoirs to prevent lipid aggregates from entering the microfluidic network and blocking the flow. Finally, the PDMS chip was bonded to a glass coverslip (Type 1, Assistent, Germany) by exposing the surfaces being bonded to an Oxygen plasma (10 W plasma power, $10 \mathrm{~s}$ exposure, $25 \mathrm{sccm}$, plasma etcher from Diener, Royal Oak, MI) and then binding the two exposed surfaces together to create a sealed microfluidic device. This was placed in an oven at $60{ }^{\circ} \mathrm{C}$ for 10 mins to enhance the adhesion.

\section{Formation of Giant Unilamellar Vesicles}

Giant unilamellar vesicles (GUVs) were created by electroformation using a Nanion Vesicle Prep Pro setup. $60 \mu \mathrm{l}$ of $5 \mathrm{mg} / \mathrm{ml}$ 1,2-diphytanoyl-sn-glycero-3-phosphocholine (DPhPC) lipid (Avanti Polar Lipids) in chloroform was spread on the conducting surface of an Indium Tin Oxide (ITO) coated glass slide (Nanion/Visiontek) within a rubber O-ring. The chloroform was evaporated for $10 \mathrm{mins}$ in a desiccator following which $600 \mu \mathrm{l}$ of the appropriate buffer $(200 \mathrm{mM}$
Sucrose in a $5 \mathrm{mM}$ Phosphate Buffer for $\mathrm{pH} 7$ or $200 \mathrm{mM}$ Sucrose in a $5 \mathrm{mM}$ acetic acid buffer for $\mathrm{pH}$ 5) was deposited within the O-ring and a sandwich made with another ITO coated slide (conducting surfaces facing each other). This was placed in the Nanion Vesicle Prep Pro whereupon electroformation proceeds in 3 steps: (i) The a/c voltage increases linearly from 0 to $3 \mathrm{~V}$ peak to peak (p-p) at $5 \mathrm{~Hz}$ in 5 mins. (ii) The voltage stays at $3 \mathrm{~V} \mathrm{p}-\mathrm{p}$ and $5 \mathrm{~Hz}$ for $2 \mathrm{hrs}$. (iii) The voltage decreases linearly to $0 \mathrm{~V}$ at $5 \mathrm{~Hz}$ in $5 \mathrm{mins}$. The electroformation was carried out at $37.5^{\circ} \mathrm{C}$. The vesicles were stored at $4{ }^{\circ} \mathrm{C}$ and used within a week.

\section{Microfluidic flow control}

The microfluidic flows were controlled by applying suction at the outlet reservoir using a neMESYS syringe pump system with a $1 \mathrm{ml}$ Duran Borosilicate glass syringe (ILS, Germany). The tubing used was Upchurch 1520G (0.03 inch inner diameter). At the inlets, pipette tips with $50 \mu \mathrm{l}$ of vesicle stock solution and $50 \mu \mathrm{l}$ of $2 \mathrm{mM}$ norfloxacin solution respectively were input into the two reservoirs, using a previously described technique $^{25}$. Initially, to ensure a uniform distribution of norfloxacin throughout the network, a fast flow was applied (approx. $200 \mu \mathrm{l} / \mathrm{hr}$ flow rate). Once the fluorescence intensities were observed to be uniform in the detection regions, the flow rate was decreased to about 5-10 $\mu \mathrm{l} / \mathrm{hr}$. Data collection was started once the flows settled and individual vesicles were slow enough $(\sim 0.8 \mathrm{~mm} / \mathrm{s})$ to be tracked in the field of view $(120 \mu \mathrm{m}$ across) for about 5-10 frames to ensure a reliable statistical analysis of the relevant measured quantities. It is important to note that we study individual vesicles as they pass through the network- this is not a bulk experiment - with a throughput up to 100 vesicles per hour.

\section{Data Analysis}

We determined normalised fluorescence intensity differences between the exterior and interior of the vesicles at $t=0\left(\Delta I_{l}\right)$ and later at $t_{f}\left(\Delta I_{2}\right)$ :

$$
\begin{array}{cc}
\Delta I_{1}=\frac{I_{\text {out }}-I_{1}}{I_{\text {out }}} \quad(t=0) \\
\Delta I_{2}=\frac{I_{\text {out }}-I_{2}}{I_{\text {out }}} \quad\left(t=t_{f}\right)
\end{array}
$$

$I_{1}$ and $I_{2}$ refer to the average internal fluorescence intensities measured around the centre of the vesicles at times $t=0$ and $t$ $=t_{f}$ and $I_{\text {out }}$ refers to the fluorescence intensity outside the vesicle, which remains constant. After solving the diffusion equation (for full details of the theoretical model and MATLAB image analysis see supplementary information), we obtained the following equation that was used for analysis:

$$
\ln \left(\Delta I_{2}-\Delta I_{1}+1\right)=-3 \frac{P t}{R}
$$


where $P$ is the permeability coefficient, $R$ the vesicle radius and $t$ the time taken to travel from the initial to the final vesicle detection point.

\section{Results and discussion}

The final microfluidic chip design is the result of a number of preliminary investigations. Preliminary single vesicle experiments implied that the time scale of norfloxacin diffusion through vesicle membranes was on the order of minutes at $\mathrm{pH}$ 7. Furthermore, microfluidics experiments revealed a vesicle speed of around $0.8-1 \mathrm{~mm} / \mathrm{s}$ to be the optimum for detection in our system, as mentioned above. Based on these considerations, we designed a network of microfluidic channels which the vesicles would take around 5-6 minutes to traverse. At these flow speeds, our preliminary experiments indicated that the norfloxacin mixes across the entire channel width within a couple of millimetres' distance from the $\mathrm{T}$ junction. In order to ensure that this was the case at the initial $t=0$ detection point in our system, we chose the length to this point (from the $\mathrm{T}$ junction) to be significantly longer $(\sim 20 \mathrm{~mm})$ in the final design.

To assess the diffusion through the membrane, we measure the intensity within the vesicles at different points in the chip. Figure 2 shows data for vesicles at $\mathrm{pH} 5$ and $\mathrm{pH}$ 7. The microscopy images show vesicles (dark) in the microfluidic channels at different points, surrounded by the autofluorescing norfloxacin. Data is shown for two lengths travelled, $L=45.5$ $\mathrm{mm}$ and $L=182 \mathrm{~mm}$; data for other lengths is presented in the supplementary information. From the images it is clear that vesicles at $\mathrm{pH} 5$ remain dark as they travel through the network, whereas at $\mathrm{pH} 7$ they become progressively brighter. Thus the autofluorescing norfloxacin permeates the membrane at $\mathrm{pH} 7$ far more readily than at $\mathrm{pH} 5$. This effect is further apparent in the plots in Figure 2. At pH 7 (red circles), as the length travelled increases, $I_{i n}$ increases and there is a corresponding decrease in $\Delta I$. Almost no significant change is observed at $\mathrm{pH}$ 5 (green triangles). The radial dependence of $\Delta I$ at $t=0$ (black squares) occurs since our measurements are in brightfield rather than confocal mode; at later times, the radial dependence is both due to this effect as well as due to drug diffusion. This is addressed in our analysis.

Figure 3 shows three different techniques used to analyse the data and determine the permeability coefficient of norfloxacin. The equation atop Figure $3 \mathrm{a}$ can be extracted from the theoretical analysis (supplementary information). Since the process being studied is diffusion, we expect to see an exponential dependence of $I_{\text {in }}$ on time (and hence length $L$ ). This analysis requires values of vesicle velocity and radius, for which the average velocity $(0.81 \pm 0.01 \mathrm{~mm} / \mathrm{s})$ and average radius $(13.6 \pm 0.1 \mu \mathrm{m})$ measured $(\mathrm{pH} 7)$ were used. Using these and the data from the exponential fit, we obtain a permeability coefficient ( $\mathrm{pH} 7$ ) of $P=5.2 \pm 0.4 \times 10^{-7} \mathrm{~cm} / \mathrm{s}$.

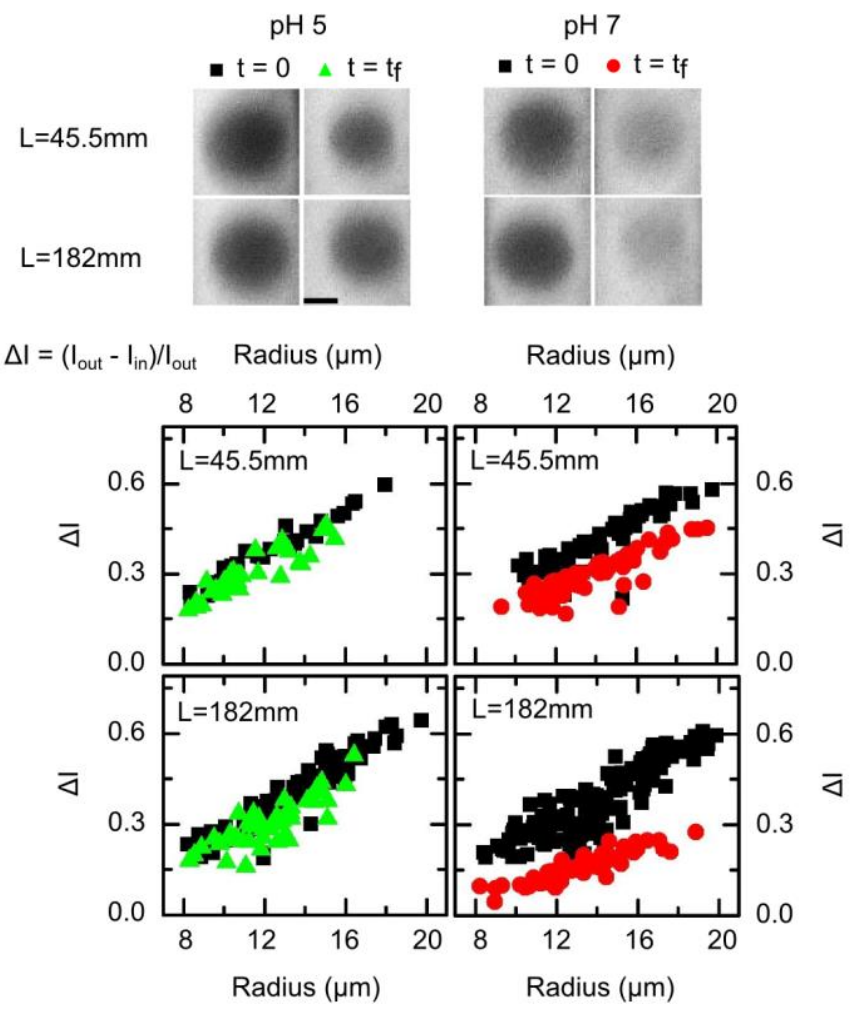

Figure 2. Norfloxacin diffusion into single lipid vesicles. Images of autofluorescent norfloxacin diffusing into vesicles $\left(\lambda_{e x}=340 \mathrm{~nm}\right)$ at $\mathrm{pH} 5$ (left) and $\mathrm{pH} 7$ (right). $\mathrm{L}$ is the length travelled from the initial $(t=0)$ to the final $\left(t=t_{f}\right)$ vesicle detection point. It is evident that there is an increase in the fluorescence intensities inside the vesicles $\left(l_{\text {in }}\right)$ at $\mathrm{pH} 7$, and a corresponding decrease in $\Delta l$. The decrease in $\Delta l$ is proportional to the length ( $L$ ) travelled, as seen in the plots (the difference between the black and red points becomes larger as $L$ increases). At pH 5, the decrease in $\Delta l$ is much less apparent (the green and black points overlap) and $l_{\text {in }}$ shows a much smaller increase within the timescales measured. It is thus clear that the permeability of norfloxacin through the lipid bilayer is much higher at $\mathrm{pH} 7$ than at $\mathrm{pH}$ 5. The scale bar is $10 \mu \mathrm{m}$ (in all images). Each data point in the plots represents a measurement on an individual vesicle. The radial dependence in the plots is discussed in the text and in further detail in the supplementary information.

Furthermore, since we determine the flow speed for each vesicle, we can calculate the time taken by each vesicle to travel the length $L$ from the initial to the final detection point. Collating this with our measurements of $\Delta I$ and the radius of each vesicle (an average of the semi-major and semi-minor axes), we know all the parameters required to determine the permeability coefficients for each vesicle detected using equation (1). Histograms of these values for the $\mathrm{pH} 7$ experiments are shown in Figure 3c. The average permeability coefficient determined is $P=5.9 \pm 0.2 \times 10^{-7} \mathrm{~cm} / \mathrm{s}(N=272)$. Finally, we can use the same equation (1) to determine the permeability coefficient $(\mathrm{pH} 7)$ from the slope of Figure $3 \mathrm{~b}$. This gives $P=6.6 \pm 0.6 \times 10^{-7} \mathrm{~cm} / \mathrm{s}$. The difference in value between the average (Figure 3c) and the slope (Figure 3b) can be attributed to the standard linear regression (least squares) 


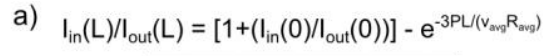

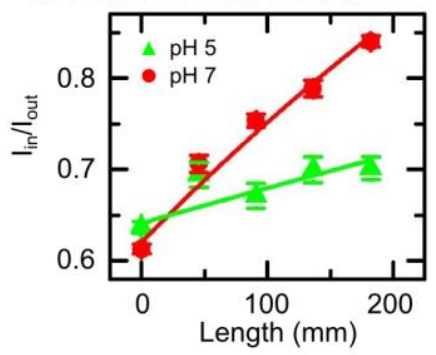

b)

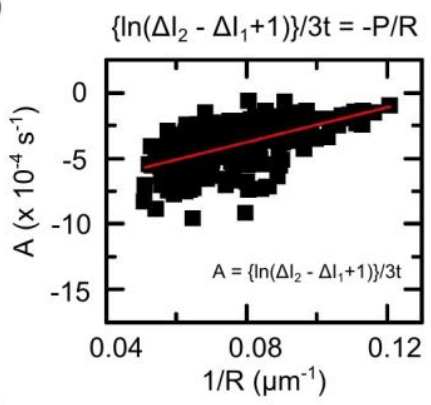

c)

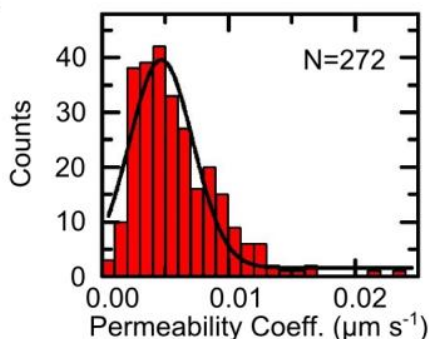

Figure 3. Measurements of the permeability coefficient $(P)$. a. Dependence of $I_{\text {in }} / I_{\text {out }}$ with respect to the length $L$ travelled by vesicles from the initial to the final detection point. The solid lines are fits based on our theoretical expectation of a diffusive process governing the transport of norfloxacin across lipid membranes. Error bars represent standard errors of the mean. From the fit, we have extracted $P=5.2 \pm 0.4 \times 10^{-7} \mathrm{~cm} / \mathrm{s}$ at $\mathrm{pH}$ 7. $b$. Dependence of parameter $A$ (defined in the figure) on $1 / R$. The slope of the linear fit gives us another technique of determining $P$. The value obtained ( $p H$ 7) was $P=6.6 \pm 0.6 \times 10^{-7} \mathrm{~cm} / \mathrm{s}$. c. Histogram of $P$ values for individual vesicles. Values determined using equation (1). The average value of these single vesicle calculations gives $P=5.9 \pm 0.2 \times 10^{-7} \mathrm{~cm} / \mathrm{s}(\mathrm{N}=272)$.

technique employed in determining the slope. All errors reported are standard errors of the mean. Thus there is good agreement using all three analysis techniques.

At $\mathrm{pH} 5$, the majority of the norfloxacin molecules are positively charged, but at $\mathrm{pH} 7$ a significant proportion of the molecules exist in their uncharged form ${ }^{20}$. As indicated in the plots in Figure 2 and from Figure 3a, it is clear that at $\mathrm{pH} 5$ fewer norfloxacin molecules permeate the membrane. This is as expected, since polar charged molecules have a low affinity for the non-polar core of the bilayer. From our data we estimate a permeability coefficient of $0.5-1.5 \times 10^{-7} \mathrm{~cm} / \mathrm{s}$ at $\mathrm{pH} 5$, a 6 -fold decrease as compared to $\mathrm{pH}$ 7. This also confirms that our vesicles are indeed a stable model system not susceptible to leakage.
With fluoroquinolones there is significant discrepancy among the published values of apparent partition coefficients, and hence in the predicted permeability coefficients ${ }^{16}$. Apparent partition coefficients for norfloxacin have been reported as 0 $(\mathrm{pH} \mathrm{7})^{21}, 0.003(\mathrm{pH} \mathrm{7.2})^{20}$ and $0.01(\mathrm{pH} \mathrm{7.2})^{14}$, which would imply a permeability coefficient ranging from 0 to around $10^{-5}$ $\mathrm{cm} / \mathrm{s}$. Our method does not require knowledge of the partition coefficient; we provide a direct measurement of the permeability coefficient, and the values we obtain are well within the range predicted in the literature ${ }^{20}$.

We thus have a new and direct technique of measuring the permeability coefficient of drugs passively diffusing across lipid membranes. In contrast to previously described methods, we do not require any labelling or chemical complex formation in order to track the drug molecules. We further do not need to determine the drug partition coefficients in artificial aqueousorganic phase systems; we directly obtain the permeability coefficient across the membrane of interest, which is the quantity required when designing and testing new drugs.

At present the experiment throughput is limited primarily by the camera speed, since faster flows (vesicle speeds $>1.5$ $\mathrm{mm} / \mathrm{s}$ ) lead to a blurring of the vesicles in the images. The throughput could be increased by using higher flow rates with a faster camera, enabling the detection of a larger number of vesicles in a shorter period of time.

Our technique paves the way to assess antibiotic diffusion in different vesicle compositions, including proteo-liposomes containing membrane channels (such as $\mathrm{OmpF}^{26}$ ) implicated in antibiotics transport. Scaling down the microfluidic channel dimensions should even allow the examination of individual bacteria and a similar assay could be used to quantify the permeability of drugs in resistant and normal pathogens.

This label-free technique is directly applicable when the drug or molecule being studied is autofluorescent. Many interesting biomolecules and drugs are indeed autofluorescent, especially in the UV, and thus give this assay a wide applicability. However, if the molecule of interest is not autofluorescent, one would have to tailor the experiment to suit the study, for example by using reporter complexes within the vesicles that fluoresce in the presence of the drug, as demonstrated in reference [10].

Membrane permeation across artificial and cell membranes has been extensively studied in microfluidic platforms $s^{9,10,27-31}$. However, most of the work has concentrated on developing platforms for supported lipid bilayers in microfluidic chambers. As discussed in the Introduction, lipid vesicles are geometrically much better cell mimics, and techniques for studying permeation across vesicle membranes can directly be adapted to studying permeation into living cells. Work has previously been done on using vesicles in microfluidic platforms to study membrane permeation ${ }^{9,10}$, but the techniques 
involved fixing or trapping vesicles, which will limit throughput. Our assay involves analysing flowing vesicles in free solution, and is thus capable of much higher throughput. Furthermore, the microfluidic chip fabrication techniques involved are simpler, since structures or chemical modifications for vesicle trapping are not required. We trust that these simplifications make our assay accessible and useful to not only the microfluidics community, but also to scientists involved in drug development and biologists interested in permeation studies on the single cell level.

\section{Conclusions}

We have described a novel, label-free technique of studying passive drug transport across lipid membranes that directly determines drug permeability coefficients using lipid vesicles. It is experimentally simple and can be adapted for cell work. The experiment itself is robust and can be run for hours at a stretch. As a proof-of-principle, we have used it to determine the permeability coefficient of the antibiotic norfloxacin across DPhPC lipid membranes at $\mathrm{pH} 7$, and have validated predictions for the effect of $\mathrm{pH}$ on norfloxacin permeability.

\section{Acknowledgements}

This work was supported by a European Research Council (ERC) grant (261101 PassMembrane) to UFK. JC acknowledges support from an Internal Graduate Studentship, Trinity College, Cambridge. CC is supported by the ERC. SP acknowledges the support of the Leverhulme Trust and the Newton Trust through an Early Career Fellowship. AJ is supported by the Mexican National Council of Science and Technology. We thank Thomas Muller for help with the lithography and Tuomas Knowles for the use of his lithography facilities.

\section{Notes and references}

${ }^{a}$ Biological and Soft Systems, Dept. of Physics, Univ. of Cambridge, Cavendish Laboratory, JJ Thomson Avenue, Cambridge CB3 OHE, United Kingdom. E-mail: ufk20@cam.ac.uk Phone: +44 (0) 1223337007 Fax: +44 (0) 1223337000

* These authors contributed equally to this work.

Electronic Supplementary Information (ESI) available: [AutoCAD file of mask design, MATLAB analysis scripts, AVI file of typical experiment and Supplementary Information document]. See DOI: 10.1039/b000000x/

1. Editorial, Nature, $2013, \mathbf{4 9 5}, 141$

2. M. Mckenna, Nature, 2013, 499, 394-396.

3. H. K. Allen, J. Donato, H. H. Wang, K. A. Cloud-Hansen, J. Davies and J. Handelsman, Nature Reviews Microbiology, 2010, 8, 251-259.

4. J-M Pagès, C. E James and M. Winterhalter, Nature Reviews Microbiology, 2008, 6, 893-903.

5. G. M. Whitesides, Nature, 2006, 442, 368-373.

6. M. T. Guo, A. Rotem, J. A. Heyman and D. A. Weitz, Lab on a Chip, 2012, 12, 2146-2155.

7. A. E. Ekpenyong, G. Whyte, K. Chalut, S. Pagliara, F. Lautenschläger, C. Fiddler, S. Paschke, U. F. Keyser, E. R. Chilvers and J. Guck, PLoS ONE, 2012, 7 (9): e45237
8. D. R. Gossett, W. M. Weaver, N. S. Ahmed and D. Di Carlo, Annals of Biomedical Engineering, 2010, DOI: 10.1007/s10439010-0199-8.

9. T. Robinson, P. Kuhn, K. Eyer and P. S. Dittrich, Biomicrofluidics, 2013, 7, 044105.

10. P. Kuhn, K. Eyer, S. Allner, D. Lombardi and P. S. Dittrich, Analytical Chemistry, 2011, 83, 8877-8885.

11. L. S. Matchette, A. Agrawal and T. J. Pfefer, Photochemistry and Photobiology, 2007, 83, 1386-1393.

12. K. Drlica and X. Zhao, Microbiology and Molecular Biology Reviews, 1997, 61, 377-392.

13. J. M. Diver, L. J. V. Piddock and R. Wise, Journal of Antimicrobial Chemotherapy, 1990, 25, 319-333.

14. K. Hirai, H. Aoyama, T. Irikura, S. Iyobe and S. Mitsuhashi, Antimicrobial Agents and Chemotherapy, 1986, 29 (3), 535-538.

15. J. S. Chapman and N. H. Georgopapadakou, Antimicrobial Agents and Chemotherapy, 1988, 32 (4), 438-442.

16. P. G. S. Mortimer and L. J. V. Piddock, Journal of Antimicrobial Chemotherapy, 1991, 28, 639-653.

17. G. E. Flaten, A. B. Dhanikula, K. Luthman and M. Brandl, European Journal of Pharmaceutical Sciences, 2006, 27, 80-90.

18. K. Mandagere, T. N. Thompson and K-K. Hwang, Journal of Medicinal Chemistry, 2002, 45, 304-311.

19. M. Kansy, F. Senner and K. Gubernator, Journal of Medicinal Chemistry, 1998, 41, 1007-1010.

20. H. Nikaido and D. Thanassi, Antimicrobial Agents and Chemotherapy, 1993, 37, 1393-1399.

21. J. Ashby, L. J. V. Piddock and R. Wise, Journal of Antimicrobial Chemotherapy, 1985, 16, 805-808.

22. Edelstein, N. Amodaj, K. Hoover, R. Vale and N. Stuurman, Current Protocols in Molecular Biology, 2010, 92, 14.20.114.20.17.

23. D. Qin, Y. Xia and G. M. Whitesides, Nature Protocols, 2010, 5, 491-502.

24. G. M. Whitesides, E. Ostuni, S. Takayama, X. Jiang and D. E. Ingber, Annual Review of Biomedical Engineering, 2001, 3, 335373.

25. M. H. Horrocks, L. Rajah, P. Jönsson, M. Kjaergaard, M. Vendruscolo, T. P. J. Knowles and D. Klenerman, Analytical Chemistry, 2013, 85, 6855-6859.

26. K. R. Mahendran, M. Kreir, H. Weingart, N. Fertig and M. Winterhalter, Journal of Biomolecular Screening, 2010, 15, 302307.

27. S. Ota, H. Suzuki and S. Takeuchi, Lab on a Chip, 2011, 11, 2485-2487.

28. T. Osaki, Y. Watanabe, R. Kawano, H. Sasaki and S. Takeuchi, Journal of Microelectromechanical Systems, 2011, 20 (4), 797799.

29. E. W. K. Young, M. W. L. Watson, S. Srigunapalan, A. R. Wheeler and C. A. Simmons, Analytical Chemistry, 2010, 82, 808-816.

30. T. Nisisako, S. A. Portonovo and J. J. Schmidt, Analyst, 2013, 138, 6793-6800.

31. I. Hutter, E. Muller, P. M. Kristiansen, S. Kresak and L. Tiefenauer, Microfluid Nanofluid, 2013, 14, 421-429. 